\title{
Ökonomisierung der Pflege - Formalisierung und Prekarisierung von Pflegearbeit
}

In der feministischen Wohlfahrtsstaatsforschung spielt die Auseinandersetzung mit „care“, also Sorgearbeit, eine wichtige Rolle. Im Vordergrund stehen dabei meistens die Kinderbetreuung und die informelle Sorgearbeit. Dieser Beitrag rückt einen anderen Ausschnitt der Sorgearbeit in den Mittelpunkt: die formelle Pflegearbeit in ambulanten Diensten und Pflegeheimen, die im Zuge demografischer Veränderungen, modernisierter Geschlechterrollen und pluralisierter Familienformen sowie zunehmender, oft berufsbedingter Mobilitätsanforderungen an Bedeutung gewonnen hat und weiter gewinnen wird. Analysiert wird der Wandel dieses traditionell weiblichen Berufsfeldes im Kontext von Formalisierung, Professionalisierung und Prekarisierung.

DIANA AUTH

\section{Einleitung}

Trotz heftiger öffentlicher Proteste vonseiten der Opposition, der Pflegeverbände, der Krankenkassen sowie der Arbeitgeberverbände ist von der konservativ-liberalen Koalition im Herbst 2012 eine weitere, eher kleine Pflegereform verabschiedet worden. Danach gibt es nun staatliche Zuschüsse zur privaten Pflegevorsorge (der sog. „Pflege-Bahr“), und Demenzkranke erhalten als Vorgriff auf den neuen Pflegebedürftigkeitsbegriff verbesserte Leistungen, die durch eine Beitragssatzsteigerung um 0,1 \% gegenfinanziert werden. Mit diesem „Reförmchen“ werden die demografischen und sozioökonomischen Probleme, die auf die Pflegeversicherung in den nächsten Jahrzehnten zukommen - insbesondere die pflegepolitischen Folgen des gesellschaftlichen Alterungsprozesses, des Rückgangs des Erwerbspersonenpotenzials und der Modernisierung der Geschlechterverhältnisse nicht zu bewältigen sein. Denn es ist absehbar, dass die Zahl der Pflegebedürftigen ansteigen wird, während gleichzeitig das häusliche Pflegepotenzial zurückgeht - unter anderem aufgrund der gestiegenen Frauenerwerbstätigkeit, veränderter pflegekultureller Einstellungen und beruflicher Mobilitätsanforderungen. Um das entstehende care deficit (Hochschild 1995) zu beheben, muss die bislang größtenteils von Frauen geleistete informelle Pflegearbeit neu organisiert und verteilt werden. Dabei wird die formelle Pflegearbeit einen entscheidenden Anteil haben.
Im Folgenden werden der Wandel der staatlichen und der unternehmerischen Regulierung der formellen Pflegearbeit und seine Folgen in den Blick genommen. Dies geschieht auf der Basis einer systematischen Auswertung amtlicher und sonstiger Statistiken und Berichte, politischer und akteursbezogener Dokumente sowie wissenschaftlicher Studien zum Themenfeld. Abschnitt 2 untersucht, wie sich die staatliche Regulierung der formellen Pflege, die in hohem Maße von Ökonomisierungsprozessen geprägt ist, seit der Einführung der Pflegeversicherung Mitte der 1990er Jahre gewandelt hat (Outputs). Unter Ökonomisierung in der Pflegebranche wird hier ein Prozess der Integration effizienzorientierter Prinzipien in die Organisation pflegebezogener Leistungen, der Rückgang öffentlicher Tätigkeiten im Pflegebereich durch Auslagerung auf andere Leistungsanbieter (Pluralisierung von Trägern und Anbieterkonkurrenz), die Auslagerung staatlicher Pflegeleistungen auf private Anbieter, die keinerlei Beziehungen zum Staat aufweisen sowie die Stärkung der Macht der Pflegebedürftigen durch die Gewährung von mehr Wahlfreiheit („Konsumentensouveränität“) verstanden (Pfau-Effinger et al. 2008; Auth 2012a). ${ }^{1}$

1 Die Ökonomisierungsprozesse können dazu führen, dass nicht mehr die Versorgung der kranken oder pflegebedürftigen Menschen im Zentrum steht, sondern der wirtschaftliche Gewinn, der mit ihnen erzielt werden kann (Verkehrung der Mittel-Zweck-Beziehung nach Kühn (2004)). 
In Abschnitt 3 stehen die längerfristigen Auswirkungen (Outcomes) auf die formelle Pflegearbeit im Vordergrund, die durch Regulierungen auf der Unternehmensebene geprägt sind. Die Formalisierung oder De-Privatisierung, also die Expansion beruflicher Pflegearbeit auf Kosten der informellen, kann dabei unterschiedliche Formen annehmen. Es geht darum zu untersuchen, wie sich die Anteile unqualifizierter (angelernte Pflegekräfte), semi-professioneller (ausgebildete Pflegekräfte) und professioneller Pflegearbeit (Absolventinnen und Absolventen pflegewissenschaftlicher Studiengänge) verschoben haben. ${ }^{2}$ Im nächsten Schritt (4) werden die geschlechtsspezifischen Outcomes der pflegepolitischen Reformen und der unternehmerischen Regulierung analysiert. Abschließend (5) werden die Outcomes im Bereich der formellen Pflegearbeit daraufhin untersucht, ob ein Trend in Richtung Prekarisierung erkennbar ist.

Unter Prekarisierung wird hier ein Prozess verstanden, der drei Dimensionen aufweist: In der ersten Dimension wird der Wandel der Struktur von Beschäftigungsverhältnissen in den Blick genommen. Die Prekarisierung von Beschäftigung(sverhältnissen) zeigt sich an der Unterschreitung oder Absenkung der in der Pflegebranche bestehenden Normalitätsstandards. Dies bezieht sich auf die materielle Absicherung (nicht-existenzsicherndes Einkommen), auf die sozial(versicherungsrechtlich)e Absicherung (fehlende oder defizitäre Integration in das soziale Sicherungssystem), auf die Beschäftigungsstabilität (unterbrochene Beschäftigung) sowie auf die betriebliche Integration und gewerkschaftliche Mitbestimmung (kein Betriebsrat, keine Tarifbindung) (Mayer-Ahuja 2003; Keller/Seifert 2007). ${ }^{3}$ Zum Zweiten zeigt sich Prekarisierung auch innerhalb von Beschäftigungsverhältnissen durch Qualitätsverschlechterungen in Bezug auf die Arbeitsorganisation, die Arbeitsinhalte und die Arbeitsbedingungen. Arbeitsplatzunsicherheit, Arbeitsverdichtung und die Entgrenzung von Erwerbsarbeit sind Kennzeichen „prekäre[r] Normalarbeit" (Jürgens 2011, S. 380). Die Prekarisierung von Beschäftigung und Erwerbsarbeit wirkt sich in der dritten Dimension auch auf „das restliche Leben“ aus, vor allem auf die Planbarkeit, die gesellschaftliche Teilhabe und die Möglichkeiten einer selbstbestimmten Lebensführung. Unter dem Stichwort der „Reproduktionskrise“ hat Jürgens (2010) die Prekarisierung der Selbst-Reproduktion von Personen, die über die Vereinbarkeitsprobleme von Beruf und Familie deutlich hinausgeht, beschrieben. Dabei geht es bei der prekären Selbstsorge um die institutionelle Überforderung von Menschen, die vielfach in Demotivation, Identitätskrisen, Erschöpfungszuständen und Krankheit mündet (vgl. auch Jürgens 2011).

\section{Regulierung der Pflegearbeit seit Mitte der 1990er Jahre}

\subsection{Einführung der Pflegeversicherung}

Im Jahr 1995 trat nach 20 Jahren kontroverser Diskussion die Pflegeversicherung als fünfte Säule des deutschen Sozialversicherungssystems in Kraft. Seitdem ist Pflegebedürftigkeit als soziales Risiko institutionell über eine beitragsfinanzierte Pflichtversicherung abgesichert, wobei ein stark körper- und tätigkeitsbezogenes Pflegebedürftigkeitskonzept institutionalisiert wurde. Die Pflegebedürftigen können zwischen einem Pflegegeld und/oder ambulanten Sachleistungen oder stationärer Pflege wählen. Im Unterschied zur Krankenversicherung, an deren Struktur sich die Pflegeversicherung orientiert, ist die Pflegeversicherung nur eine „pauschalierte Grundsicherung“ (Landenberger 1994, S. 326) oder Teilkaskoabsicherung, allerdings ohne Bedürftigkeitsprüfung. Es gelten weiterhin der Vorrang der Selbsthilfe und das Subsidiaritätsprinzip, was konkret bedeutet, dass die Pflegebedürftigen oftmals Zuzahlungen zu ambulanter und stationärer Pflege leisten müssen. Um die $\mathrm{Zu}$ zahlungen zu finanzieren, muss zunächst das eigene Einkommen und Vermögen sowie das der Ehepartner und Kinder verwendet werden, bevor die „Hilfe zur Pflege“ greift (Gerlinger/Röber 2009; Roth 2000; Pfau-Effinger et al. 2008). Mit der Einführung der Pflegeversicherung wurden mehrere Ziele verfolgt: Erstens sollten die Sozialhilfeträger durch die Versicherungslösung von den enorm gestiegenen Kosten für stationär versorgte Pflegebedürftige entlastet werden. Zweitens sollte die infrastrukturelle Versorgung der Pflegebedürftigen quantitativ und qualitativ verbessert werden, vor allem im ambulanten Bereich. Aus Wettbewerbsgründen wurden in diesem Kontext zum einen private und freigemeinnützige Anbieter gleichgestellt, zum anderen dürfen mehr Pflegedienste und -heime eine Zulassung erhalten als erforderlich sind. Der Ausbau ambulanter Dienste diente auch dem dritten Ziel, der Stärkung der kostengünstigeren häuslichen Pflege. Der in der Pflegeversicherung geltende Vorrang der häuslichen Pflege ( $\$ 3$ SGB XI) sowie das Prinzip „ambulant vor stationär“ ( $\$ 43$ SGB XI) werden

\footnotetext{
2 Zur Entwicklung personenbezogener Dienstleistungen allgemein vgl. Gottschall (2008).

3 Um das Ausmaß der Prekarität zu erfassen, muss zudem zum einen die Haushaltslage betrachtet, zum anderen eine Lebenslaufperspektive eingenommen werden (Keller/Seifert 2007). Da es hier aber weniger um die prekäre Lebenssituation einzelner Beschäftigter auf der Mikroebene geht, sondern um die Prekarisierungstendenzen professioneller Pflegearbeit auf gesamtgesellschaftlicher Ebene, also die Prozesse und Entwicklungen, die durch politische und unternehmerische Regulierungen hervorgerufen werden, bleiben diese beiden Aspekte in dieser Abhandlung weitgehend unberücksichtigt.
} 
einerseits durch die Zahlung eines Pflegegeldes, andererseits durch die Finanzierung ambulanter Sachleistungen (auch als Kombinationsleistung) unterstützt. Dabei wurden die kostendeckenden Pflegesätze durch Leistungskomplexe, in denen bestimmten Tätigkeiten standardisierte Zeiteinheiten und Preise zugeordnet sind, abgelöst. Durch diese Zweigleisigkeit - Pflegegeld und Sachleistungen - wird sowohl die informelle Pflege aufgewertet als auch die formelle Pflege gefördert (Landenberger 1994; Roth 2000; Pfau-Effinger et al. 2008).

Mit der Pflegeversicherung hat einerseits eine Ausweitung von Leistungen stattgefunden, andererseits wurden ökonomische Prinzipien eingeführt. Im Kontext der Globalisierung und des Standortwettbewerbs wurden seit den 1990er Jahren auch im Bereich des Wohlfahrtsstaates Elemente von Marktlogik und Effizienz etabliert, so auch im Gesundheitswesen. In Deutschland sind insbesondere mit der Standardisierung und Pauschalierung der Leistungen sowie mit der Schaffung eines Anbieterwettbewerbs durch die Gleichstellung privater Träger mit den Wohlfahrtsverbänden effizienzorientierte kostensenkende Maßnahmen verankert worden. Demgegenüber hat - anders als beispielsweise in Großbritannien - kein Privatisierungsprozess im Sinne der zunehmenden Kostenverlagerung auf die Pflegehaushalte stattgefunden. Die Konsumentensouveränität wurde durch die Wahl zwischen verschiedenen pflegerischen Leistungen und Anbietern gestärkt (Auth 2012a).

\subsection{Pflegepolitische Reformen}

Die ersten pflegepolitischen Reformen erfolgten im Jahr 2002 und dienten dem quantitativen und qualitativen Ausbau der formellen Pflege. Zum einen wurde das Pflege-Qualitätssicherungsgesetz eingeführt, das ambulante Pflegedienste und Pflegeheime verpflichtet, ein Qualitätsmanagement einzuhalten, in dem auch eine qualitätssichernde personelle Ausstattung berücksichtigt werden muss. Zum anderen trat das Pflegeleistungs-Ergänzungsgesetz in Kraft, mit dem erstmals der betreuungs- und beaufsichtigungsbezogene Bedarf Demenzkranker berücksichtigt wurde (Gerlinger/Röber 2009).

Mitte 2008 wurde die erste größere, von der Großen Koalition ausgehandelte Pflegereform durchgeführt. Mit dem Pflege-Weiterentwicklungsgesetz wurde einerseits versucht, die demografisch und sozio-ökonomisch bedingten Kosten der Pflege zu begrenzen. Andererseits wurden lange ausstehende Reformprojekte verwirklicht, wie die Anhebung der finanziellen Leistungen sowie die Einführung einer Leistungsdynamisierung und die stärkere Berücksichtigung von Demenzkranken durch die Erhöhung des Betreuungsbetrags. Zudem wurden bestehende pflegepolitische Ansätze und Prinzipien weiterentwickelt wie die Stärkung der häuslichen Pflege, die Förderung gemischter Pflegearrangements und die Einführung vereinbarkeitsorientierter Maßnahmen. Dazu zählen die Schaffung von Pflegestützpunkten (Anspruch auf Pflegeberatung), die Förderung der ehrenamtlichen Pflege und das Pflegezeitgesetz, wonach Beschäftigte in Betrieben mit mehr als 15 Beschäftigten den Rechtsanspruch haben, sich für maximal sechs Monate vollständig oder teilweise freistellen zu lassen, um einen nahen Angehörigen zu pflegen. Während dieser Zeit werden weder Einkommen noch Einkommensersatzleistungen gezahlt, aber es besteht weiterhin Sozialversicherungsschutz. Daran anknüpfend ist Anfang 2012 unter Familienministerin Schröder die Familienpflegezeit in Kraft getreten, der zufolge Beschäftigte ihre Arbeitszeit für maximal zwei Jahre auf bis zu 15 Stunden pro Woche reduzieren können, um einen pflegebedürftigen Angehörigen häuslich zu pflegen. Die Beschäftigten erhalten in der Pflegephase ein Einkommen, das nur um die Hälfte der Arbeitszeitreduzierung gekürzt ist. In der sogenannten Nachpflegephase wird das reduzierte Einkommen beibehalten, bis das Arbeitszeit- oder Wertguthaben wieder ausgeglichen ist. Ein Rechtsanspruch besteht allerdings nicht (Gerlinger/Röber 2009; Auth 2012b). In den pflegepolitischen Reformen stand die informelle Pflege im Mittelpunkt. Die formelle Pflege soll der Unterstützung häuslicher Pflege im Sinne gemischter Pflegearrangements dienen. Demgegenüber sollten keine Anreize zum weiteren Ausbau der teuren vollstationären Pflege geboten werden.

\section{Auswirkungen der Pflegereformen auf die formelle Pflegearbeit}

\subsection{Der Wandel der Trägerstruktur in der Pflegebranche}

Die Gleichstellung privater Dienste und Einrichtungen mit freigemeinnützigen Trägern sowie die Erzeugung eines Anbieterwettbewerbs haben die Pflegelandschaft massiv verändert. Im Jahr 2011 gibt es gut 12.300 ambulante Dienste. Knapp zwei Drittel davon werden von privaten Trägern getragen. Der Anteil der freigemeinnützigen Pflegedienste liegt 2011 bei gut einem Drittel. Der Anteil der öffentlichen Träger liegt im selben Jahr bei lediglich 1,4\%. Dass es nur wenige öffentliche Anbieter gibt, hängt mit der langen Tradition freigemeinnütziger Träger in der Altenpflege in Deutschland zusammen (Roth 2000). Verglichen mit dem Jahr 1999 ist die Zahl der ambulanten Dienste insgesamt um $14 \%$ angestiegen, wovon ausschließlich die privaten Träger profitiert haben (+41\%). Der Anteil der privaten Träger hat seit 1999 um knapp ein Viertel zugenommen, während der Anteil der freigemeinnützigen Träger im selben Umfang gesunken ist (Tabelle 1).

Der Wandel bei den Pflegeheimen verläuft in eine ähnliche Richtung. Im Jahr 2011 gibt es ebenfalls gut 12.300 Pflegeheime. Hier sind die freigemeinnützigen Träger mit $54 \%$ führend, gefolgt von den privaten Trägern mit gut $40 \%$ und den öffentlichen Trägern mit $5 \%$. Gegenüber 1999 ist die Zahl der Pflegeheime um knapp 40 \% gestiegen. Damit hat die Pflegeversicherung in deutlich höherem Maße ein 
Wachstum der stationären Pflege bewirkt als des Ausbaus der ambulanten Pflegeinfrastruktur. Davon haben die privaten Träger mit einer Steigerung um gut $60 \%$ und die freigemeinnützigen Träger - allerdings deutlich abgeschwächt - mit einer Steigerung um gut ein Drittel profitiert. Auch die Anteile haben sich seit 1999 verschoben. Der Anteil der privaten Pflegeheime ist um 16 \% gestiegen, während der Anteil der freigemeinnützigen Träger um knapp $4 \%$ gesunken ist (Tabelle 1).

Diese Entwicklungen zeigen, dass die Ziele der Pflegeversicherung, der Ausbau der Pflegeinfrastruktur und die Schaffung einer pluralen - neben den verschiedenen freigemeinnützigen nun auch private Träger umfassenden - Anbieterstruktur, erreicht worden sind (vgl. auch Kümmerling 2008). Dabei wurde die prägende Stellung der Wohlfahrtsverbände im konservativ-korporatistischen deutschen Wohlfahrtsstaat (Esping-Andersen 1990) aufgegeben. Die privaten Anbieter ambulanter Pflegedienste und Pflegeheime sind eindeutig die Gewinner des Ausbaus der formellen Pflege und der politisch initiierten Trägerkonkurrenz. Sie sind Mitte der 1990er Jahre mehr oder weniger neu gestartet und heute für gut $60 \%$ der ambulanten und $40 \%$ der stationären Pflege zuständig.

\subsection{Löhne und Lohnentwicklung}

Der Wandel der Trägerstruktur hat auch Auswirkungen auf die Löhne und die Lohnentwicklung in der Pflegebranche. Faktisch fallen weder die Beschäftigen der privaten Träger noch der Großteil der Beschäftigten kirchlicher oder sonstiger freigemeinnütziger Träger in den Geltungsbereich von Tarifverträgen. Die zahlenmäßig größte Gruppe von Beschäftigten in der Pflegebranche, die Angestellten in privaten Pflegekonzernen, arbeitet ohne Tarifvertrag, der mit einer DGB-Gewerkschaft, insbesondere mit ver.di, ausgehandelt wurde. ${ }^{4}$ Die Wohlfahrtsverbände, die zwar eigene Vereinbarungen (sog. "Dritter Weg“) geschaffen haben, ${ }^{5}$ orientierten sich lange Zeit an den Abkommen im Öffent-

\section{TABELLE 1}

Trägerstruktur in der ambulanten und stationären Pflege (1999 und 2011)

Angaben in absoluten Zahlen und in Prozent

\begin{tabular}{|c|c|c|c|c|c|c|}
\hline & \multicolumn{2}{|c|}{1999} & \multicolumn{2}{|c|}{2011} & \multicolumn{2}{|c|}{ Veränderung } \\
\hline & Anzahl & $\begin{array}{l}\text { Anteil } \\
\text { in } \%\end{array}$ & Anzahl & $\begin{array}{l}\text { Anteil } \\
\text { in } \%\end{array}$ & $\begin{array}{c}\text { Anzahl } \\
\text { in } \%\end{array}$ & $\begin{array}{c}\text { Anteil } \\
\text { in } \%\end{array}$ \\
\hline \multicolumn{7}{|l|}{ Ambulante Dienste } \\
\hline PrivateTräger & 5.504 & 50,9 & 7.772 & 62,9 & $+41,2$ & $+23,7$ \\
\hline Freigemeinnützige Träger & 5.103 & 47,2 & 4.406 & 35,7 & $-13,7$ & $-24,3$ \\
\hline Öffentliche Träger & 213 & 2,0 & 171 & 1,4 & $-19,7$ & $-29,7$ \\
\hline Gesamt & 10.820 & 100,0 & 12.349 & 100,0 & $+14,1$ & \\
\hline \multicolumn{7}{|l|}{ Stationäre Pflege } \\
\hline Private Träger & 3.092 & 34,9 & 4.998 & 40,5 & $+61,6$ & $+15,9$ \\
\hline Freigemeinnützige Träger & 5.017 & 56,6 & 6.721 & 54,4 & $+34,0$ & $-3,9$ \\
\hline ÖffentlicheTräger & 750 & 8,5 & 635 & 5,1 & $-15,3$ & $-39,3$ \\
\hline Gesamt & 8.859 & 100,0 & 12.354 & 100,0 & $+39,5$ & \\
\hline
\end{tabular}

Quelle: Statistisches Bundesamt 2001, 2013; Berechnungen der Autorin.

lichen Dienst. Aufgrund des Kostendrucks, der durch die direkte Konkurrenz mit den privaten Anbietern entstanden ist, nimmt diese Orientierung mehr und mehr ab (Kümmerling 2008). Dies geschieht zum einen durch Öffnungsklauseln und die flexible Anwendung der Arbeitsvertragsrichtlinien (AVR). ${ }^{6}$ Zum anderen können die AVR durch die Gründung von Tochtergesellschaften (Outsourcing) oder den Einsatz von Leiharbeit umgangen werden. Die Löhne und die Arbeitsbedingungen der Beschäftigten verschlechtern sich in der Folge. Die Löhne für die Beschäftigten in den privaten Pflegediensten liegen deutlich niedriger als im Öffentlichen Dienst und in den AVR. Demgegenüber sind die Löhne im Managementbereich der privaten Dienste beträchtlich höher als im pflegerischen Bereich (Theobald 2004; Burau et al. 2007).
42009 hat der im selben Jahr gegründete Arbeitgeberverband Pflege e.V. mit medsonet, einer Gewerkschaft des Christlichen Gewerkschaftsbundes, einen Tarifvertrag für Pflegehilfskräfte abgeschlossen. Nach einer Klage der Gewerkschaft ver.di haben das Arbeitsgericht Hamburg und das Landesarbeitsgericht Hamburg medsonet im Jahr 2011 bzw. 2012 für nicht tariffähig erklärt, unter anderem aufgrund der zu geringen Mitgliederzahl. Das Urteil wurde im Juni 2013 durch das Bundesarbeitsgericht bestätigt (http://justiz.hamburg.de/pressemitteilungen/2900396/ pressemeldung-2011-1.html; http://www.verdi.de/themen/ nachrichten/++co++68da0b4c-742c-11e1-42cc0019b9e321cd; Frankfurter Rundschau vom 12.06.2013).

5 So gelten bei der Caritas Arbeitsvertragsrichtlinien (AVR), die die Arbeitsrechtliche Kommission des Deutschen Caritasverbandes abgeschlossen hat. Diese Kommission ist paritätisch mit Beschäftigten und Dienstgebern besetzt; Beschlüsse brauchen in der Regel eine Dreiviertelmehrheit (http://www.caritas.de/glossare/avrtarifrechtdercaritas). Auch in der Diakonie gibt es AVR (derzeit gültig: AVR DW EKD). Die Landeskirchen können allerdings selbst entscheiden, ob sie die AVR übernehmen. Im Bereich der Diakonie gibt es zudem drei Tarifverträge mit ver.di (Diakonie Hamburg, evangelisch-lutherische Kirche Nordelbien und Evangelische Kirche Berlin-Brandenburg-schlesische Oberlausitz) (http://www.diakonie.de/arbeitsvertragsrichtlinien-dwekd-995.htm; www.verdi.de). Die Arbeiterwohlfahrt (AWO) hat einen eigenen Arbeitgeberverband gegründet, derTarifverträge mit der Gewerkschaft ver.di aushandelt. Hier gilt derzeit der Manteltarifvertrag BMT AW II. Auch die AWO-Gliederungen können frei entscheiden, ob sie sich diesem Tarifvertrag anschließen (http://www.agv-awo.de).

6 So kann z. B. von den AVR der Caritas aus wirtschaftlichen Gründen abgewichen werden, wenn die entsprechende Regionalkommission dem Antrag der Einrichtung zustimmt. 
TABELLE 2

Bruttolohnentwicklung im Öffentlichen Dienst (West) nach TVöD-B (Pflege- und Betreuungseinrichtungen) (2008-2013)

Angaben in Euro und in Prozent

\begin{tabular}{|c|c|c|c|c|c|c|}
\hline & $\begin{array}{c}\text { E 7a/Stufe } 2 \\
\text { (ausgebildete/r } \\
\text { Altenpfleger/in) }\end{array}$ & $\begin{array}{c}\text { E 9d/Stufe } 4 \\
\text { (Pflegedienst- } \\
\text { leitung) }\end{array}$ & $\begin{array}{c}\text { Veränderung } \\
\text { gegenüber } \\
\text { dem Vorjahr } \\
\text { in } \%\end{array}$ & $\begin{array}{l}\text { AVR Anlage 3a/KR 4/ } \\
\text { Stufe } 2 \text { (ausgebildete/r } \\
\text { Altenpfleger/in) }\end{array}$ & $\begin{array}{l}\text { Veränderung } \\
\text { gegenüber } \\
\text { dem Vorjahr } \\
\text { in } \%\end{array}$ & $\begin{array}{l}\text { Durchschnittliche } \\
\text { Tarifabschlüsse } \\
\text { (West) } \\
\text { in } \%\end{array}$ \\
\hline 2013 & $2.365,73$ & $3.496,68$ & $+2,8$ & $2.340,03$ & $+2,8$ & \\
\hline 2012 & $2.300,86$ & $3.400,79$ & $+3,5$ & $2.275,86$ & $+3,5$ & $+3,9$ \\
\hline 2011 & $2.223,05$ & $3.285,79$ & $+1,1$ & $2.198,90$ & $+1,1$ & $+3,0$ \\
\hline 2010 & $2.198,80$ & $3.249,94$ & $+1,2$ & $2.174,91$ & $+1,2$ & $+2,2$ \\
\hline 2009 & $2.172,73$ & $3.211,40$ & $+2,8$ & $2.149,12$ & $+4,3$ & $+2,4$ \\
\hline 2008 & $2.113,55$ & $3.123,93$ & & $2.060,52$ & & $+3,9$ \\
\hline
\end{tabular}

Quellen: http://oeffentlicher-dienst.info (TVöD 2008;TVöD-B 2009-2012; AVR 2009-2012); http://www.avrneu.de/upload/Caritas\%20AVR/Dokumente/BK_08_06.pd (AVR 2008-2009), WSI (verschiedene Jahrgänge 2008-2012): Tarifpolitischer Jahresbericht; Berechnungen der Autorin.

Um die Löhne und die Lohnentwicklung in der Pflegebranche abzubilden, werden im Folgenden die Tariflöhne im Öffentlichen Dienst sowie - exemplarisch für den freigemeinnützigen Bereich - bei der Caritas betrachtet. Im Öffentlichen Dienst ist seit 2005 der Tarifvertrag für den Öffentlichen Dienst (TVöD) in Kraft, den die Gewerkschaft ver.di mit der Tarifgemeinschaft deutscher Länder (Bund und Kommunen) ausgehandelt hat. Dieser gilt in den Pflegeeinrichtungen und Diensten der Kommunen. ${ }^{7}$

Vergleicht man die Tariflohnentwicklung im Öffentlichen Dienst (TVöD-B Pflege- und Betreuungseinrichtungen) in Westdeutschland und in den AVR der Caritas mit den durchschnittlichen Tariferhöhungen eines Jahres, so zeigt sich, dass die Bruttolöhne von Kranken- und Altenpflegerinnen bzw. -pflegern sowie von Pflegedienstleitungen mit Ausnahme des Jahres 2009 in geringerem Maße angestiegen sind als die durchschnittlichen Tariferhöhungen, die in dem jeweiligen Jahr abgeschlossen und in Kraft getreten sind (Tabelle 2).

Die Löhne für ausgebildete Altenpflegende in Pflegeeinrichtungen (nach ein bzw. zwei Jahren beruflicher Tätigkeit) liegen nach den AVR der Caritas leicht unter den Löhnen im Öffentlichen Dienst. ${ }^{8}$ Die Lohnsteigerungen sind mit Ausnahme des Jahres 2009, als die AVR neu ausgehandelt wurden, identisch (Tabelle 2).
Der WSI-Lohnspiegel für Pflegeberufe ${ }^{9}$ aus dem Jahr 2012 hat ergeben, dass der durchschnittliche Bruttomonatsverdienst einer Altenpflegerin/eines Altenpflegers bei $2.148 €$ liegt. Der Median beträgt $2.032 €$, d. h., das untere Viertel der Altenpflegenden verdient weniger als $1.714 €$, das obere Viertel mehr als $2.412 €$. Der durchschnittliche Bruttomonatsverdienst der in der Altenpflege Tätigen liegt damit unterhalb des TVöD bzw. den AVR der Caritas, was man unter anderem auf die niedrigeren Löhne in den privaten Pflegediensten und -einrichtungen zurückführen kann. In der Studie wird dementsprechend auch festgestellt, dass Beschäftigte in Pflegeberufen (zu denen neben der Altenpflege auch die krankenpflegerischen Berufe gehören), die in tarifgebundenen Betrieben arbeiten, deutlich mehr verdienen. Der Unterschied liegt bei ca. $480 €$ brutto. Von den befragten Altenpflegenden geben nur $39 \%$ an, in einem tarifgebundenen Betrieb zu arbeiten (Bispinck et al. 2012).

Seit Mitte 2010 gibt es in der Pflegebranche - gemeint ist die Altenpflege und die ambulante Krankenpflege - einen Branchenmindestlohn, der nach dem Entsendegesetz für allgemeinverbindlich erklärt wurde. Den Anstoß dazu gaben die Gewerkschaft ver.di und die Arbeiterwohlfahrt (AWO), die 2008 den Antrag zur Aufnahme der Altenpflege in das Arbeitnehmerentsendegesetz stellten, dem sich die Vereinigung der kommunalen Arbeitgeberverbände
7 Vor 2005 galt der Bundesangestelltentarif (BAT), der auch eine Sondertabelle „Kr" für Krankenpflege umfasste. Die erste Entgelttabelle für den TVöD-B Pflege- und Betreuungseinrichtungen gibt es 2008. Aufgrund der Unterschiede zwischen BAT undTVöD ist es an dieser Stelle nicht möglich, zwischen den beiden Tarifvertragswerken sinnvolle Vergleiche herzustellen. Generell gilt, dass junge Beschäftigte mit Berufserfahrung besser gestellt werden, während sich die Arbeitsbedingungen älterer Beschäftigter mit Kindern tendenziell verschlechtert haben.
8 Die Löhne für Altenpflegerinnen/Altenpfleger nach den AVR der Diakonie sind ähnlich.

9 Im WSI-Lohnspiegel wird eine Analyse der Einkommensund Arbeitsbedingungen von Beschäftigten in Pflegeberufen vorgenommen. Die Auswertung bezieht sich auf über 3.500 Datensätze aus einer Onlinebefragung der Jahre 2006-2012. Zu den Pflegeberufen zählen sämtliche Berufe in der Kranken- und Altenpflege (Bispinck et al. 2012). 
anschloss. Anlass der Diskussion war die Arbeitnehmerfreizügigkeit in der Europäischen Union (EU), die 2011 begann und aufgrund derer man, wenn es keinen Mindestlohn gibt, mit negativen Auswirkungen auf die Altenpflegebranche rechnete. Der 2010 vereinbarte Mindestlohn gilt auch für ausländische Betriebe, die Arbeitnehmer in Deutschland beschäftigen. Er beträgt seit Mitte $2013 \mathrm{im}$ Westen $9 €$ und im Osten $8 €$. Davon betroffen sind ca. 800.000 Beschäftigte, die überwiegend in der Grundpflege arbeiten. Darunter fallen auch Leiharbeiter und geringfügig Beschäftigte. Ausgeschlossen sind nur Auszubildende und Praktikanten (WSI 2011; https://gesundheit-soziales.verdi. de/branchenpolitik/pflegeeinrichtungen/mindestlohn).

Die pluralisierte, von den privaten Trägern dominierte Trägerstruktur hat zur Enttariflichung der Pflegebranche beigetragen. Mittlerweile überwiegen individualvertragliche Abkommen (Theobald 2004; Burau et al. 2007). Weniger als die Hälfte der in der Altenpflege Beschäftigten erhält tarifliche oder daran angelehnte Löhne. Das Lohnniveau in privaten Diensten und Heimen liegt unterhalb des Niveaus staatlicher und freigemeinnütziger Träger. Die freigemeinnützigen Träger haben sich dem Lohnabsenkungstrend durch neue und flexibel anwendbare Entlohnungssysteme angepasst (Kümmerling 2008). Das pflegepolitische Ziel der Kostensenkung wurde demnach erreicht. Die Abwärtsspirale der Löhne konnte lediglich durch den Mindestlohn aufgehalten werden, mit dem verhindert wird, dass die Löhne von Vollzeitbeschäftigten in der Pflegebranche unter das Existenzminimum und damit erkennbar in den Bereich prekärer Beschäftigung rutschen. Die Analyse der Lohnentwicklung zeigt weiter, dass die Lohnzuwächse in der Altenpflege geringer ausgefallen sind als im gesamtgesellschaftlichen Durchschnitt. Zusammenfassend lässt sich sagen, dass das traditionelle deutsche Beschäftigungsmodell mit flächendeckenden Branchentarifverträgen und ausgebauten betrieblichen Mitbestimmungsrechten in der Altenpflege niemals angekommen ist. Hier herrscht stattdessen ein „fragmentierte[s] deutsche[s] Beschäftigungsmodell“ (Kümmerling 2008, S. 137) vor.

\subsection{Entwicklung der Beschäftigungs- und Qualifikationsstrukturen}

Der Arbeitsmarkt Pflege ist seit der Einführung der Pflegeversicherung kontinuierlich am Wachsen. Mit der Zunahme ambulanter und stationärer Pflege ist auch die Zahl der beruflich Pflegenden angestiegen. In den ambulanten Pflegediensten arbeiten im Jahr 2011 knapp 290.000 Beschäftigte. In den Pflegeheimen sind gut 660.000 Personen beschäftigt. Die Zahlen sind seit 1999 um die Hälfte angestiegen (Tabelle 3). Der Beschäftigungsanstieg wird etwas relativiert, wenn man die Vollzeitäquivalente betrachtet. Dann beträgt der Anstieg zwischen 2003 und 2011 bei den ambulanten Pflegediensten nur gut $44 \%$ und bei den Pflegeheimen sogar nur ca. $23 \%$ (Statistisches Bundesamt 2005; 2013). ${ }^{10}$ Aufgrund des gesellschaftlichen Alterungsprozesses wird der Arbeits- kräftebedarf weiter steigen, während sich bereits jetzt ein Arbeitskräftemangel in der Pflegebranche abzeichnet.

Die Zunahme der Beschäftigung in der Pflegebranche zwischen 1999 und 2011 ging mit einem Rückgang des Anteils an Vollzeitbeschäftigten von $31 \%$ auf $27 \%$ in den ambulanten Diensten und von $48 \%$ auf knapp ein Drittel in den Pflegeheimen einher. Demgegenüber ist der Anteil der Teilzeitbeschäftigungen und der Mini-Jobs enorm angestiegen. Zugenommen haben vor allem Teilzeitbeschäftigungen mit einer Arbeitszeit von über 50 \%. Die Zahl dieser Teilzeitbeschäftigungen ist in den ambulanten Diensten zwischen 1999 und 2011 um über $100 \%$ und in den Pflegeheimen sogar um über 130 \% gestiegen. Damit arbeiten im Jahr 2011 gut ein Drittel der Beschäftigten in Pflegediensten und -heimen „lange Teilzeit“. Zählt man noch diejenigen Teilzeitbeschäftigten dazu, die weniger als $50 \%$, aber nicht geringfügig beschäftigt sind (ca. $15 \%$ ), so arbeitet ungefähr die Hälfte der Beschäftigen in der Pflegebranche auf Teilzeitbasis. Zwischen 1999 und 2011 hat auch die Zahl der Mini-Jobs in der Pflegebranche zugenommen: in ambulanten Pflegediensten um über $50 \%$ und in den Pflegeheimen um $45 \%$. Der Anteil der Mini-Jobs hat sich dagegen kaum verändert und liegt bei gut einem Fünftel in den ambulanten Diensten und bei knapp einem Zehntel in den Pflegeheimen (Tabelle 3).

In der Pflegebranche ist nicht nur der Anteil der Teilzeitbeschäftigen, sondern auch der Anteil der Befristungen hoch. Laut WSI-Lohnspiegel sind ca. ein Viertel der Altenpflegerinnen/Altenpfleger und knapp ein Drittel der Altenpflegehelferinnen/Altenpflegehelfer befristet beschäftigt (Bispinck et al. 2012).

Neben der zunehmenden Formalisierung hat infolge der Einführung der Pflegeversicherung auch eine (Semi-) Professionalisierung von Pflegearbeit stattgefunden. Die Zahl der Pflegefachkräfte in ambulanten Diensten ist zwischen 1999 und 2011 um 68 \% und in Pflegeheimen um $56 \%$ angestiegen. Der Anteil der Pflegefachkräfte in ambulanten Diensten hat sich im selben Zeitraum von 48 auf 51 \% erhöht, in den Pflegeheimen leicht von 31 auf $32 \%$. Nicht nur die Zahl und der Anteil der Pflegefachkräfte haben zugenommen, auch die Akademisierung der Pflege ist vorangeschritten. Hatten im Jahr 1999 nur 808 Beschäftigte in Pflegeheimen einen pflegewissenschaftlichen (Fach-) Hochschulabschluss, so waren es 2011 bereits 2.870 , also gut dreimal so viele. In den ambulanten Pflegediensten ist die Entwicklung ähnlich: Im Jahr 1999 wiesen gut $420 \mathrm{Be}-$ schäftigte einen pflegewissenschaftlichen (Fach-)Hochschulabschluss auf, im Jahr 2011 waren es mit 1.080 bereits mehr als doppelt so viele. An dieser Stelle sollte allerdings auch erwähnt werden, dass der Anteil derjenigen ohne pflegerische Qualifikation in Pflegeheimen über $40 \%$ beträgt (Tabelle 4).

10 Die Umrechnung in Vollzeitäquivalente existiert erst ab der Pflegestatistik 2003. 
TABELLE 3

\section{Entwicklung der Voll- und Teilzeitbeschäftigung in der Pflegebranche (1999 und 2011)}

Angaben in absoluten Zahlen und in Prozent

\begin{tabular}{|c|c|c|c|c|c|c|}
\hline & \multicolumn{2}{|c|}{1999} & \multicolumn{2}{|c|}{2011} & \multicolumn{2}{|c|}{ Veränderung } \\
\hline & Anzahl & $\begin{array}{l}\text { Anteil } \\
\text { in } \%\end{array}$ & Anzahl & $\begin{array}{l}\text { Anteil } \\
\text { in } \%\end{array}$ & $\begin{array}{l}\text { Anzahl } \\
\text { in } \%\end{array}$ & $\begin{array}{l}\text { Anteil } \\
\text { in } \%\end{array}$ \\
\hline \multicolumn{7}{|l|}{ Ambulante Dienste } \\
\hline Vollzeitbeschäftigte & 56.914 & 31,0 & 79.755 & 27,4 & $+40,1$ & $-11,4$ \\
\hline \multicolumn{7}{|l|}{ Teilzeitbeschäftigte... } \\
\hline ...über $50 \%$ & 49.149 & 26,7 & 100.514 & 34,6 & $+104,5$ & $+29,3$ \\
\hline $\begin{array}{l}\text {..5 } 50 \% \text { und weniger, aber } \\
\text { nicht geringfügig beschäftigt }\end{array}$ & 28.794 & 15,7 & 42.487 & 14,6 & $+47,6$ & $-6,7$ \\
\hline ...geringfügig & 39.126 & 21,3 & 61.671 & 21,2 & $+57,6$ & $-0,4$ \\
\hline Sonstige* & 9.799 & 5,3 & 6.287 & 2,2 & $-35,8$ & $-59,4$ \\
\hline Personal insgesamt & 183.782 & 100 & 290.714 & 100 & $+58,2$ & \\
\hline \multicolumn{7}{|l|}{ Pflegeheime } \\
\hline Vollzeitbeschäftigte & 211.544 & 48,0 & 212.416 & 32,1 & 0,4 & $-33,1$ \\
\hline \multicolumn{7}{|l|}{ Teilzeitbeschäftigte... } \\
\hline ...über $50 \%$ & 100.897 & 22,9 & 241.000 & 36,5 & $+138,9$ & $+59,3$ \\
\hline $\begin{array}{l}\ldots 50 \% \text { und weniger, aber } \\
\text { nicht geringfügig beschäftigt }\end{array}$ & 54.749 & 12,4 & 101.863 & 15,4 & $+86,1$ & $+24,1$ \\
\hline ...geringfügig & 42.795 & 9,7 & 62.371 & 9,4 & $+45,7$ & $-2,8$ \\
\hline Sonstige* & 30.955 & 7,0 & 43.529 & 6,6 & $+41,1$ & $-5,9$ \\
\hline Personal insgesamt & 440.940 & 100 & 661.179 & 100 & $+50,0$ & \\
\hline
\end{tabular}

* Praktikant/in, Schüler/in, Auszubildende/r, Helfer/in im freiwilligen sozialen

Jahr und Zivildienstleistende.

Quelle: Statistisches Bundesamt 2001, 2013; Berechnungen der Autorin.

Trotz niedriger Löhne und unterdurchschnittlicher Lohnentwicklung stellte die Einführung der Pflegeversicherung einen entscheidenden Schubfaktor für die Formalisierung und (Semi-)Professionalisierung der Pflegebranche dar. Die Pflegeberufe wurden gestärkt und gegenüber der medizinischen Profession aufgewertet (Landenberger 1994). Beispielsweise wirken Pflegefachkräfte zusammen mit den Ärztinnen und Ärzten des Medizinischen Dienstes der Krankenversicherungen (MDK) bei der Feststellung des Grades der Pflegebedürftigkeit und damit der Leistungszuteilung mit. Und ambulante Pflegedienste erhalten nur dann einen Versorgungsvertrag, wenn eine ausgebildete Pflegefachkraft in leitender Position eingesetzt ist. Auch in stationären Einrichtungen obliegt die Verantwortung für die Pflege ausgebildeten Pflegefachkräften. Die Schaffung eines klar umgrenzten, unabhängigen Berufsfeldes für Pflegefachkräfte und „the creation of some independence $v i s-\grave{a}$ - $v i s$ the dominating medical profession was a novelty in the German health care system" (Burau et al. 2007, S. 146, Herv. i.O.). Einen Anteil an der gestiegenen Fachkraftquote, vor allem in Bereich der ambulanten Dienste, und an dem Bedarf an (Fach-)Hochschulabsolventen haben auch die pflegepolitischen Regulierungen im Rah- men der Qualitätssicherung (Pflegereform 2002). In den Rahmenverträgen auf Landesebene werden seitdem Personalrichtwerte festgesetzt. Zudem ist in der Heimpersonalverordnung eine Fachkraftquote von mindestens $50 \%$ vorgeschrieben (Gerlinger/Röber 2009), die allerdings oftmals nicht eingehalten wird (vgl. Tabelle 4). Des Weiteren nimmt die Zahl der Pflegefachkräfte zu, weil infolge der Einsparungen im Krankenhausbereich seit über einem Jahrzehnt Krankenpflegepersonal im Altenpflegebereich, vor allem in der ambulanten Pflege, unterkommt (Kümmerling 2008; Gottschall 2008).

Es zeichnen sich also insgesamt widersprüchliche Tendenzen im Hinblick auf die Beschäftigungs- und Qualifikationsstrukturen in der Pflegebranche ab. Einerseits findet mit der Ausweitung ambulanter Dienste und Pflegeheime eine Formalisierung, (Semi)-Professionalisierung und Akademisierung der Pflegeberufe statt, andererseits erfolgt der Ausbau der Pflegeinfrastruktur vorwiegend auf der Basis von Teilzeitbeschäftigungsverhältnissen („lange Teilzeit“) und - wenn auch in geringerem Maße - von Mini-Jobs. Das hat vermutlich damit zu tun, dass Unternehmen bei Teilzeitarbeitsplätzen niedrigere Löhne ${ }^{11}$ und eine höhere Flexibilität kalkulieren. Kaum berücksichtigt wird dabei, dass der administrative Aufwand und die Arbeitsübergaben ansteigen, was die Arbeitszufriedenheit der Pflegenden und die Pflegequalität negativ beeinflussen kann. Im Kontext des Fachkräftemangels wird Pflegediensten und -heimen geraten, Arbeitszeitaufstockungen und Vollzeitarbeitsplätze anzubieten (Schulz 2012).

\subsection{Entwicklung der Arbeitsbedingungen}

Der Wettbewerb zwischen den Trägern und die Anpassung der Strategien der freigemeinnützigen an die privaten Träger beeinflussen auch die Arbeitsbedingungen in der Pflegebranche negativ. Zwei Langzeitstudien belegen, dass sich die Arbeitssituation der beruflich Pflegenden seit Einführung der Pflegeversicherung verschlechtert hat, vor allem durch Personalabbau, der zu Zeitdruck, verringerten Entscheidungsspielräumen und einem erhöhten Arbeitsvolumen geführt hat (Zimber et al. 1999; Hollmann/Schmidt 1999; Zellhuber 2003). Die schlechten Arbeitsbedingungen im Pflegebereich werden in vielen Studien aufgezeigt (vgl. Zusammenstellungen der Studien in: Zimber 1998 und Gläser/Höge 2005). Eine aktuelle Studie zu Arbeitsbedingungen im Pflegeberuf im europäischen Vergleich zeigt, dass weniger als $50 \%$ der befragten Beschäftigen in Pflegeheimen und -diensten mit ihrer Arbeitssituation zufrieden ist. Gründe für die Unzufriedenheit des Pflegepersonals sind hohe körperliche und

11 Die Bruttostundenlöhne von teilzeitbeschäftigten Frauen in Westdeutschland liegen 2,50 € niedriger als die Löhne vollzeitbeschäftigter Frauen, so das Ergebnis einer Studie auf der Basis der Daten des Sozio-oekonomischen Panels (SOEP) (Wolf 2010). 
Personal in ambulanten Pflegediensten und Pflegeheimen nach Berufsabschluss (1999 und 2011)

Angaben in absoluten Zahlen und in Prozent

\begin{tabular}{|c|c|c|c|c|c|c|}
\hline & \multicolumn{2}{|c|}{1999} & \multicolumn{2}{|c|}{2011} & \multicolumn{2}{|c|}{ Veränderung } \\
\hline & Anzahl & $\begin{array}{l}\text { Anteil } \\
\text { in } \%\end{array}$ & Anzahl & $\begin{array}{l}\text { Anteil } \\
\text { in } \%\end{array}$ & $\begin{array}{l}\text { Anzahl } \\
\text { in } \%\end{array}$ & $\begin{array}{l}\text { Anteil } \\
\text { in } \%\end{array}$ \\
\hline \multicolumn{7}{|l|}{ Ambulante Dienste } \\
\hline Pflegefachkraft*... & 88.840 & 48,3 & 150.003 & 51,6 & $+68,8$ & $+6,7$ \\
\hline $\begin{array}{l}\text {... davon mit pflegewissenschaftlicher } \\
\text { Ausbildung (FH/Uni) }\end{array}$ & 420 & & 1.080 & & $+157,1$ & \\
\hline $\begin{array}{l}\text { Sonstige pflegerische, sozialpädagogische oder } \\
\text { hauswirtschaftliche Qualifikation** }\end{array}$ & 41.933 & 22,8 & 63.663 & 21,9 & $+51,8$ & $-4,0$ \\
\hline Sonstige oder keine Qualifikation*** & 53.009 & 28,8 & 77.048 & 26,5 & $+45,3$ & $-8,1$ \\
\hline Gesamt & 183.782 & 100 & 290.714 & 100 & $+58,2$ & \\
\hline \multicolumn{7}{|l|}{ Pflegeheime } \\
\hline Pflegefachkraft*... & 136.252 & 30,9 & 213.458 & 32,3 & $+56,7$ & $+4,5$ \\
\hline $\begin{array}{l}\text {... davon mit pflegewissenschaftlicher } \\
\text { Ausbildung (FH/Uni) }\end{array}$ & 808 & & 2.870 & & $+255,2$ & \\
\hline $\begin{array}{l}\text { Sonstige pflegerische, sozialpädagogische oder } \\
\text { hauswirtschaftliche Qualifikation** }\end{array}$ & 91.011 & 20,6 & 159.351 & 24,1 & $+75,1$ & $+16,8$ \\
\hline Sonstige oder keine Qualifikation*** & 213.677 & 48,5 & 288.370 & 43,6 & $+35,0$ & $-10,8$ \\
\hline Gesamt & 440.940 & 100 & 661.179 & 100 & $+49,9$ & \\
\hline
\end{tabular}

* Altenpfleger/innen, Gesundheits- und Krankenpfleger/innen, Gesundheits- und Kinderkrankenpfleger/innen, Heilerzieher/innen und Heilerziehungspfleger/innen Abschluss einer pflegewissenschaftlichen Ausbildung an einer FH oder Universität.

** Krankenpflegehelfer/innen, Altenpflegehelfer/innen, Heilerziehungspflegehelfer/innen, Heilpädagoge/in, Ergotherapeut/in, Physiotherapeut/in, sonstiger Abschluss im Bereich der nichtärztlichen Heilberufe, sozialpädagogischer/sozialarbeiterischer Berufsabschluss, Familienpfleger/in, Dorfhelfer/in, sonstiger pflegerischer Beruf, Fachhauswirtschafter/in für ältere Menschen, sonstiger hauswirtschaftlicher Berufsabschluss.

*** sonstiger Berufsabschluss, ohne Berufsabschluss/noch in Ausbildung.

Quelle: Statistisches Bundesamt 2001, 2013; Berechnungen der Autorin.

emotionale Belastungen, soziale Spannungen und Konflikte in den Diensten und Einrichtungen, die schlechte Arbeitszeitorganisation und die schlechte Vereinbarkeit des Berufs mit familiären Belangen. Jede und jeder vierte Beschäftigte denkt häufig über einen Einrichtungswechsel nach, über $15 \%$ denken sogar mehrfach in der Woche über einen Berufsausstieg nach. In Alten- und Pflegeheimen ist das Arbeitsaufkommen und damit die zeitliche Belastung der Beschäftigten im internationalen Vergleich sehr hoch. Über $40 \%$ der Befragten in der Pflegebranche leiden unter Burnout (Simon et al. 2005; Gerlinger/Röber 2009). Auch im Rahmen des WSI-Lohnspiegels im Pflegebereich wurde die Arbeitszufriedenheit abgefragt. Sie liegt etwas unter dem Durchschnittswert aller Beschäftigten. Besonders unzufrieden sind die Beschäftigten in der Pflegebranche mit der Bezahlung, der Vereinbarkeit von Familie und Beruf sowie der verfügbaren Freizeit. Auch die Stressbelastung, vor allem die physische Erschöpfung, liegt deutlich über dem Durchschnitt aller Beschäftigten (Bispinck et al. 2012).

Infolge der Ökonomisierung der Pflege haben sich demnach die Arbeitsbedingungen verschlechtert. Pfau-Effinger et al. (2008) sprechen in Bezug auf die in hohem Maße normierte und standardisierte professionelle Pflegearbeit sogar von einer „Quasi-Taylorisierung“. Die neuen Manage- mentkonzepte sind auf Rationalisierung und Kostensenkung ausgelegt. Dabei entsteht ein Widerspruch zwischen den neuen Ansprüchen an Professionalisierung und höhere Qualitätsstandards (Stichworte: Zertifizierung, Technisierung und neue pflegebezogene Organisationskonzepte) und den Mängeln in der elementaren Versorgung sowie der Vernachlässigung der seelischen Bedürfnisse der Pflegebedürftigen (z. B. Wundliegen oder fehlende Zeiten für Gespräche). Die letzten beiden Entwicklungen sind durch die effizienzorientierten Maßnahmen verursacht. Die Pflege im Rahmen der Pflegeversicherung, die auf einem stark körperund tätigkeitsbezogenen Pflegebegriff basiert, lässt kaum Zeit für die sozialen Aspekte des Pflegens. Die professionell Pflegenden erfahren eine Intensivierung ihrer Arbeit durch ausgedünnte Personaldecken (Senghaas-Knoblauch 2008).

\section{Zunahme formeller Pflegearbeit bei konstant hohem Frauenanteil}

Die informelle Pflege dominiert zwar in Deutschland noch, doch es existiert seit Jahren ein deutlicher Trend zur 
Pflegebedürftige nach Art der Versorgung (1999 und 2011)

Angaben in absoluten Zahlen und in Prozent

\begin{tabular}{|c|c|c|c|c|c|c|}
\hline & \multicolumn{2}{|c|}{1999} & \multicolumn{2}{|c|}{2011} & \multicolumn{2}{|c|}{ Veränderung } \\
\hline & Anzahl & $\begin{array}{l}\text { Anteil } \\
\text { in } \%\end{array}$ & Anzahl & $\begin{array}{l}\text { Anteil } \\
\text { in } \%\end{array}$ & $\begin{array}{l}\text { Anzahl } \\
\text { in } \%\end{array}$ & $\begin{array}{l}\text { Anteil } \\
\text { in } \%\end{array}$ \\
\hline $\begin{array}{l}\text { Pflegebedürftige zu Hause versorgt, } \\
\text { davon... }\end{array}$ & 1.442 .880 & 71,6 & 1.758 .321 & 70,3 & $+21,9$ & $-1,8$ \\
\hline ... allein durch Angehörige* & 1.027 .591 & 51,0 & 1.182 .057 & $47,3 * *$ & $+15,0$ & $-7,3$ \\
\hline ...durch ambulante Pflegedienste & 415.289 & 20,6 & 576.264 & 23,0 & $+38,8$ & $+11,8$ \\
\hline Pflegebedürftige in Heimen & 573.211 & 28,4 & 743.120 & 29,7 & $+29,6$ & $+4,5$ \\
\hline Insgesamt & 2.016 .091 & & 2.501 .441 & & $+24,1$ & \\
\hline
\end{tabular}

* Entspricht den Empfänger/innen von ausschließlich Pflegegeld nach § 37 SGB XI. Empfänger/innen von Kombinationsleistungen nach

$\S 38 \mathrm{SGB} X \mathrm{I}$ sind dagegen in den ambulanten Pflegediensten enthalten.

** Der Anstieg der Pflegegeldempfänger/innen ist laut Statistischem Bundesamt im Jahr 2011 zu hoch ausgewiesen (um ca. 90.000 Personen)

die Vergleichbarkeit zu den Jahren davor ist daher eingeschränkt.

Quelle: Statistisches Bundesamt 2001, 2013; Berechnungen der Autorin.

Zunahme ambulanter und stationärer Pflege - und zwar trotz verschiedener Push- und Pull-Faktoren in Richtung informelle Pflege. Die Pflegeversicherung bietet zum einen starke Anreize für informell Pflegende, indem ein Pflegegeld gewährt und die pflegende Person unfall- und rentenversichert ist. Dies wird zum anderen unterstützt durch diverse Hemmschwellen gegenüber der Inanspruchnahme formeller Pflege. Dazu gehören die in der Regel zu leistenden $\mathrm{Zu}$ zahlungen der Pflegehaushalte, die Unterhaltsverpflichtungen und die standardisierten Pflegeleistungen.

Im Jahr 2011 werden über $47 \%$ der Pflegebedürftigen zuhause allein durch Angehörige gepflegt. Dieser Anteil ist seit 1999 um gut 7 \% gesunken. Demgegenüber ist der Anteil der Pflegebedürftigen, die zuhause (ausschließlich oder als Kombinationsleistung) durch ambulante Pflegedienste versorgt werden, im selben Zeitraum von einem Fünftel auf $23 \%$ gestiegen. Der Anteil der stationären Pflege ist zwischen 1999 und 2011 leicht von gut $28 \%$ auf knapp $30 \%$ angestiegen (Tabelle 5).

Es zeigt sich, dass die anfängliche „Resistenz gegenüber finanziellen Steuerungsversuchen" (Evers 1997, S. 517) in Richtung ambulanter Pflege im Laufe der Zeit abgemildert werden konnte. Umgekehrt traten Mitte der 1990er Jahre geäußerte Befürchtungen einer „(Re-)Privatisierung des Pflegerisikos“ (Landenberger 1994, S. 331) nicht ein. Ursachen der Zunahme formeller Pflege sind die Gewährung ambulanter und stationärer Pflegeleistungen durch die Pflegeversicherung, aber auch die Zunahme der weiblichen Erwerbsbeteiligung und sich wandelnde pflegekulturelle Einstellungen in Richtung moderner Geschlechterrollenvorstellungen (Blinkert/Klie 2004).

Trotz der Zunahme formeller Pflegearbeit hat sich an den Geschlechteranteilen kaum etwas verändert. Von den gut 950.000 Beschäftigen in ambulanten Diensten und Pflegeheimen sind im Jahr 201188 \% bzw. 84 \% weiblich. Der
Frauenanteil hat sich in der Pflegebranche infolge der Einführung der Pflegeversicherung fast nicht verändert. Trotz positiver absoluter Zuwachsraten hat der Anteil der Männer in den ambulanten Diensten sogar um $17 \%$ abgenommen (Tabelle 6).

Die Ursachen dieser weitgehenden Konstanz der Geschlechteranteile liegen in den Spezifika der Pflegearbeit, die traditionell zu den weiblich konnotierten Berufsfeldern gehört. Bis ins 20. Jahrhundert hinein pflegten „Schwestern“ als „Liebesdienste“ kranke und pflegebedürftige Menschen, und die Pflege als Tätigkeit wurde mit weiblicher Fürsorglichkeit, Geduld, Einfühlsamkeit und Empathie verknüpft (Senghaas-Knobloch 2008, S. 227f.). Pflegearbeit weist daher auch die meisten der - zumeist negativen - Merkmale traditionell weiblicher Beschäftigung auf: niedrige Löhne, schlechte soziale Absicherung, hoher Teilzeitanteil und schlechte Arbeitsbedingungen. Lediglich hinsichtlich der Arbeitsplatzsicherheit und der beruflichen Aufstiegschancen hat sich die Lage etwas verbessert. Formelle Pflegearbeit passt sich demnach gut in das modernisierte Versorgermodell ein (Pfau-Effinger 2000), in dem die weibliche Zuverdienerin über den männlichen Haupternährer abgesichert ist.

\section{Fazit: Prekarisierung trotz Formali- sierung und Professionalisierung}

Die Einführung der Pflegeversicherung stellt zunächst einmal eine Leistungsausweitung für die Pflegebedürftigen und ihre pflegenden Angehörigen dar. Trotz Zuzahlungen findet keine Privatisierung, sondern eine "partielle Sozialisierung“ (Scheiwe 2010, S. 131) des Pflegerisikos statt. Das ist insofern bemerkenswert, als die Pflegeversicherung in einer Phase 
eingeführt wurde, in der der neoliberale Um- und Abbau wohlfahrtsstaatlicher Leistungen bereits in einigen europäischen Ländern, wie Großbritannien und Schweden, im Gange war. Bei den Ökonomisierungselementen, die im Rahmen der Pflegeversicherung eingeführt wurden, handelt es sich zum einen um die Pauschalierung und Standardisierung der Leistungen, zum anderen um die Konkurrenz verschiedener Anbieter sozialer Dienste und Einrichtungen. Infolge dieser bewussten politischen „Steuerung durch Markt- und Wettbewerbsmechanismen" (Gerlinger/Röber 2009, S. 23) hat ein Anbieterwettbewerb stattgefunden, der die Pflegelandschaft in Deutschland stark verändert hat. Der unaufhaltsame Aufstieg privater Träger hat negative Auswirkungen auf die Entwicklung von Löhnen, Beschäftigungsstrukturen und Arbeitsbedingungen in der beruflichen Pflege. Es zeigen sich vielfältige Prekarisierungstendenzen:

(1) Auf der Ebene der Beschäftigungsstrukturen hat die Konkurrenz freigemeinnütziger mit privaten Trägern ohne Tarifbindung zur Enttariflichung der tarifschwachen Pflegebranche geführt. Dies hatte zur Folge, dass das Lohnniveau gesunken und die Lohnentwicklung unterhalb des gesamtgesellschaftlichen Durchschnitts verlaufen ist. Der Druck auf die - sowieso schon niedrigen - Löhne in dieser traditionellen Frauenbranche hat sich deutlich erhöht und konnte nur durch den 2010 eingeführten Mindestlohn abgemildert werden. Nicht nur die Löhne, auch das Niveau der betrieblichen Mitbestimmung ist in den Betrieben ohne Tarifbindung eher gering. Zudem ist der Ausbau der Beschäftigungsverhältnisse in sozialen Diensten und Einrichtungen der Pflegebranche in hohem Maße auf der Basis von Teilzeitbeschäftigungen (und zum Teil auch von Mini-Jobs) erfolgt. Der Anteil der sozialversicherungspflichtigen Vollzeitbeschäftigungsverhältnisse („Normalarbeitsverhältnis-

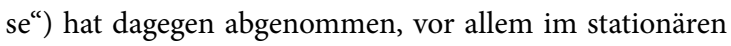
Bereich. Die in der Pflegebranche dominierenden Teilzeitbeschäftigungen gehen meist nicht mit existenzsichernden Löhnen und Renten oberhalb der Armutsgrenze einher. Das gilt in noch stärkerem Maße für Mini-Jobs. Dass die individuellen Einkommen oft prekär sind, bedeutet jedoch nicht zwangsweise, dass die beruflich Pflegenden (Frauen) in Armut leben oder nur durch die Kombination mehrerer Jobs materiell prekären Verhältnissen entfliehen können. Vermutlich handelt es sich häufig um zuverdienende (Ehe-) Frauen, die über ihren (Ehe-)Mann materiell und sozial abgesichert sind. Abgesehen von vergeschlechtlichten Berufsbildern und dem geschlechtsspezifischen Berufswahlverhalten lässt sich der konstant hohe Frauen- bzw. der niedrige Männeranteil in der Pflegebranche insbesondere vor dem Hintergrund dieses modernisierten Versorgermodells erklären. Auch hinsichtlich der Beschäftigungsstabilität ist die Lage in der Pflegebranche prekär, da ein Viertel der Beschäftigten befristet beschäftigt ist. Dieser hohe Anteil wird lediglich dadurch abgemildert, dass Pflegekräfte auf dem Arbeitsmarkt gesucht werden (Fachkräftemangel), sodass das Risiko der Arbeitslosigkeit vergleichsweise gering ist.
TABELLE 6

\section{Entwicklung des Personals in der Pflegebranche nach Geschlecht (1999 und 2011)}

Angaben in absoluten Zahlen und in Prozent

\begin{tabular}{|c|c|c|c|c|c|c|}
\hline & \multicolumn{2}{|c|}{1999} & \multicolumn{2}{|c|}{2011} & \multicolumn{2}{|c|}{ Veränderung } \\
\hline & Anzahl & $\begin{array}{c}\text { Anteil } \\
\text { in } \%\end{array}$ & Anzahl & $\begin{array}{c}\text { Anteil } \\
\text { in } \%\end{array}$ & $\begin{array}{c}\text { Anzahl } \\
\text { in } \%\end{array}$ & $\begin{array}{c}\text { Anteil } \\
\text { in \% }\end{array}$ \\
\hline \multicolumn{7}{|l|}{ Ambulante Dienste } \\
\hline Frauen & 156.398 & 85,1 & 254.665 & 87,6 & $+62,8$ & $+2,9$ \\
\hline Männer & 27.384 & 14,9 & 36.049 & 12,4 & $+31,6$ & $-16,8$ \\
\hline Personal insgesamt & 183.782 & 100 & 290.714 & 100 & $+58,2$ & \\
\hline \multicolumn{7}{|l|}{ Pflegeheime } \\
\hline Frauen & 371.271 & 84,2 & 554.068 & 83,8 & $+49,2$ & $-0,5$ \\
\hline Männer & 69.669 & 15,8 & 107.111 & 16,2 & $+53,7$ & $+2,5$ \\
\hline Personal insgesamt & 440.940 & 100 & 661.179 & 100 & $+49,9$ & \\
\hline
\end{tabular}

Quelle: Statistisches Bundesamt 2001, 2013; Berechnungen der Autorin.

(2) Auch die Bedingungen innerhalb der Erwerbsarbeit - als zweite Dimension von Prekarisierung - haben sich durch die pflegepolitischen Ökonomisierungsprozesse verschlechtert. Mit der Einführung eines körper- und tätigkeitsbezogenen Pflegebedürftigkeitsbegriffs, gestiegenen Anforderungen an die Pflegedokumentation und standardisierten Pflegeleistungen sowie der Schaffung eines Anbieterwettbewerbs, der in dieser personalintensiven Branche in hohem Maße über die Senkung der Lohnkosten geführt wurde, haben sich die Arbeitsbedingungen, insbesondere die zeitlichen und körperlichen Belastungen der beruflich Pflegenden, verschlechtert. Ein Professionalitätsverständnis, wonach sich Pflegequalität neben der Fachlichkeit auch durch Mitmenschlichkeit auszeichnet (Senghaas-Knobloch 2008), kann unter diesen Bedingungen nur schwer umgesetzt werden.

(3) Die schlechteren Arbeitsbedingungen haben auch negative Auswirkungen auf die Reproduktionsbedingungen der beruflich Pflegenden, wie die Befragungen der Beschäftigten in ambulanten Diensten und Pflegeheimen zeigen. Als einer der häufigsten Gründe für die hohe Arbeitsunzufriedenheit werden die schlechten Bedingungen der Vereinbarkeit von Beruf und Familie genannt. Auch die hohe Krankheitsrate deutet auf prekäre Selbstsorgebedingungen hin. Aufgrund des hohen Frauenanteils und der nach wie vor bestehenden Zuweisung informeller Sorgearbeit an Frauen kumulieren die Problemlagen bei beruflich pflegenden Frauen mit Kindern und/oder kranken oder pflegebedürftigen Angehörigen.

Trotz dieser Prekarisierungsprozesse in allen drei Dimensionen hat die Neuregelung und Formalisierung der Pflege auch einige emanzipatorische Outcomes hervorgebracht: Positiv hervorzuheben ist erstens die Zunahme (semi-)professioneller Pflegearbeit - gegenüber der informellen 
Pflege einerseits und gegenüber unqualifizierter Pflegearbeit andererseits. Ein erfreulicher Prozess stellt zum Zweiten die institutionelle Aufwertung der professionellen, weiblich geprägten Pflegearbeit dar. Die Kompetenzen der Pflegefachkräfte sind gegenüber den medizinischen Professionen ausgeweitet worden. Mit dieser Entwicklung geht der Anstieg des Anteils qualifizierter Pflegefachkräfte einher. Es gilt, diesen Prozess der Professionalisierung und Akademisierung des Pflegeberufs weiterzuführen. ${ }^{12}$ Die Anstrengungen auf EU-Ebene, die Zugangsvoraussetzungen zur Pflegeausbildung anzuheben und zu vereinheitlichen, sind in diesem Kontext zu begrüßen, denn sie dienen der weiteren Aufwertung von Pflegearbeit. Dem Vorschlag des Direktors des Deutschen Instituts für angewandte Pflegeforschung e.V. (dip), Weidner, die Qualifikation, die Arbeitsbedingungen, die Vergütung und das Image der professionellen Pflege durch eine konzertierte Aktion zu verbessern (http://idw-online.de/pages/de/news457850), kann infolge der Analyse der Formalisierungs- und Prekarisierungsprozesse in diesem traditionellen Frauenberuf nur beigepflichtet werden. Ziel muss es sein, die berufliche Pflegearbeit in ihren sozialen Kontext einzubetten, also Empathie und Mitmenschlichkeit zu integrieren, und dieses Pflegekonzept aus seiner "geschlechtsspezifischen Zuordnung heraus- und allgemeiner, auch monetärer Wertschätzung zuzuführen." (Senghaas-Knobloch 2008, S. 240)

12 Gleichzeitig entsteht durch die Zunahme demenziell Erkrankter auch ein erheblicher Betreuungsbedarf, der von Alten- bzw. Krankenpflegehelferinnen und -helfern gedeckt werden kann.

\section{LITERATUR}

Auth, D. (2012a): Ökonomisierung von Pflege in Großbritannien, Schweden und Deutschland, in: Zeitschrift für Gerontologie und Geriatrie 45 (7),

S. $618-623$

Auth, D. (2012b): Betreuungsgeld und Familienpflegezeit: mehr Wahlfreiheit und bessere Vereinbarkeit?, in: femina politica 21 (1), S. 135-140

Bispinck, R./Dribbusch, H./Öz, F./Stoll, E. (2012): Einkommens- und Arbeitsbedingungen in Pflegeberufen. Eine Analyse auf Basis der WSI-Lohnspiegel-Datenbank, Arbeitspapier (7), http://www.boeckler.de/pdf/ta_lohnspiegel_pflegeberufe_2012.pdf (letzter Zugriff: 25.10.2012)

Blinkert, B./Klie, T. (2004): Gesellschaftlicher Wandel und demographische Veränderungen als Herausforderungen für die Sicherstellung der Versorgung von pflegebedürftigen Menschen, in: Sozialer Fortschritt 53 (11-12), S. 319-325 Burau, V./Theobald, H./Blank, R. H. (2007): Governing Home Care, Cheltenham

Esping-Andersen, G. (1990): The Three Worlds of Welfare Capitalism, Cambridge

Evers, A. (1997): Geld oder Dienste? Zur Wahl und Verwendung von Geldleistungen im Rahmen der Pflegeversicherung, in: WSI-Mitteilungen 50 (7),

S. $510-518$

Gerlinger, T./Röber, M. (2009): Die Pflegeversicherung, Bern

Gläser, J./Höge, T. (2005): Probleme und Lösungen in der Pflege aus Sicht der Arbeits- und Gesundheitswissenschaften: Bundesanstalt für Arbeitsschutz und Arbeitsmedizin, Dortmund

Gottschall, K. (2008): Soziale Dienstleistungen zwischen Informalisierung und Professionalisierung - oder: der schwierige Abschied vom deutschen Erbe sozialpolitischer Regulierung, in: Arbeit 17 (4), S. 254-267

Hochschild, A. R. (1995): The culture of politics: traditional, post-modern, cold-modern and warm-modern ideals of care, in: Social Politics 2 (3),

S. $333-346$

Hollmann, S./Schmidt, K.-H. (1999): Gemeinsame Ziele sind der Rede wert. Studie: Pflegefachkräfte fühlen sich ihrem Heim immer weniger verbunden, in: Heim und Pflege 30 (8), S. 350-353

Jürgens, K. (2010): Deutschland in der Reproduktionskrise, in: Leviathan 38 (4), S. $559-587$
Jürgens, K. (2011): Prekäres Leben, in: WSI-Mitteilungen 64 (8), S. 379-385, http://www.boeckler.de/wsimit_2011_08_juergens.pdf

Keller, B./Seifert, H. (2007): Atypische Beschäftigungsverhältnisse - Flexibilität, soziale Sicherheit und Prekarität, in: Dies. (Hrsg.): Atypische Beschäftigung Flexibilisierung und soziale Risiken, Berlin, S. 11-27

Kühn, H. (2004): Die Ökonomisierungstendenz in der medizinischen Versorgung, in: Elsner, G. et al. (Hrsg.): Markt versus Solidarität-Gesundheitspolitik im deregulierten Kapitalismus, Hamburg, S. 25-41

Kümmerling, A. (2008): Der lange Weg zur Professionalisierung der Altenpflege und seine (nicht?)-intendierten Folgen, in: Lehndorff, S. (Hrsg.): Abriss, Umbau, Renovierung? Studien zum Wandel des deutschen Kapitalismusmodells, Hamburg, S. 136-165

Landenberger, M. (1994): Pflegeversicherung als Vorbote eines anderen Sozialstaates, in: Zeitschrift für Sozialreform 40 (5), S. 314-342

Mayer-Ahuja, N. (2003): Wieder dienen lernen? Vom westdeutschen "Normalarbeitsverhältnis“ zu prekärer Beschäftigung seit 1973, Berlin

Pfau-Effinger, B. (2000): Kultur und Frauenerwerbstätigkeit in Europa. Theorie und Empirie des internationalen Vergleichs, Opladen

Pfau-Effinger, B./Och, R./Eichler, M. (2008): Ökonomisierung, Pflegepolitik und Strukturen der Pflege älterer Menschen, in: Evers, A./Heinze, R. G. (Hrsg.): Sozialpolitik. Ökonomisierung und Entgrenzung, Wiesbaden, S. 83-98

Roth, G. (2000): Fünf Jahre Pflegeversicherung in Deutschland: Funktionsweise und Wirkungen, in: Sozialer Fortschritt 49 (8-9), S. 184-192

Scheiwe, K. (2010): Die soziale Absicherung häuslicher Pflege über Grenzen hinweg - Rechtliche Grauzonen, (Ir-)Regularität und Legitimität, in: Scheiwe, K./Krawietz, J. (Hrsg.): Transnationale Sorgearbeit. Rechtliche Rahmenbedingungen und gesellschaftliche Praxis, Wiesbaden, S. 123-149

Schulz, E. (2012): Pflegemarkt: Drohendem Arbeitskräftemangel kann entgegengewirkt werden, in: DIW-Wochenbericht 79 (51/52), S. 3-16

Senghaas-Knoblauch, E. (2008): Care-Arbeit und das Ethos fürsorglicher Praxis unter neuen Marktbedingungen am Beispiel der Pflegepraxis, in: Berliner Journal für Soziologie 18 (2), S. 221-243

Simon, M./Tackenberg, P./Hasselhorn, H.-M./Kümmerling, A./Büscher, A./Müller, B. H. (2005): Auswertung der ersten Befragung der NEXT-Studie in

Deutschland, Wuppertal, http://www.next.uni-wuppertal.de/index.php?artikelund-berichte-1 (letzter Zugriff 25.10.2012)

Statistisches Bundesamt (2001): Kurzbericht Pflegestatistik 1999. Pflege im Rahmen der Pflegeversicherung. Deutschlandergebnisse, Bonn

Statistisches Bundesamt (2005): Pflegestatistik 2003. Pflege im Rahmen der Pflegeversicherung. Deutschlandergebnisse, Bonn

Statistisches Bundesamt (2013): Pflegestatistik 2011. Pflege im Rahmen der Pflegeversicherung. Deutschlandergebnisse, Wiesbaden

Theobald, H. (2004): Care services for the elderly in Germany. Infrastructure, access and utilisation from the perspective of different user groups: WZB, Working Group on Public Health Policy, Discussion paper SP I 2004-302, Berlin

Wirtschafts- und Sozialwissenschaftliches Institut (WSI) (verschied. Jahrgänge 2008-2012): Tarifpolitischer Jahresbericht, Düsseldorf

Wolf, E. (2010): Lohndifferenziale zwischen Vollzeit- und Teilzeitbeschäftigten in Ost- und Westdeutschland, WSI-Diskussionspapier (174), Düsseldorf

Zellhuber, B. (2003): Altenpflege - ein Beruf in der Krise? Eine empirische Untersuchung der Arbeitssituation sowie der Belastung von Altenpflegekräften im Heimbereich, Königsbrunn

Zimber, A. (1998): Beanspruchung und Stress in der Altenpflege: Forschungsstand und Forschungsperspektiven, in: Zeitschrift für Gerontologie und Geriatrie 31 (6), S. $417-425$

Zimber, A./Albrecht, A./Weyerer, S. (1999): Die Beanspruchungssituation in de stationären Altenpflege nach Einführung der Pflegeversicherung: Ergebnisse einer Verlaufsstudie, in: Zeitschrift für Arbeitswissenschaft 53 (3), S. 194-201

\section{AUTORIN}

DIANA AUTH, Dr. disc. pol., ist Wissenschaftliche Assistentin am Institut für Politikwissenschaft der Justus-Liebig-Universität Gießen. Arbeitsschwerpunkte: Vergleichende Wohlfahrtsstaatsforschung, Geschlechterforschung, Wandel der Arbeitsgesellschaft, Arbeitsmarkt-, Sozial- und Familienpolitik.

diana.auth@sowi.uni-giessen.de 